\title{
28 Research Soure \\ Basal IL-6 and S100b levels predict infarct volume in acute ischaemic stroke patients
}

Francisco Purroy ( $\square$ fpurroygarcia@gmail.com )

Biomedical Research Institute of Lleida

Joan Farre-Rodriguez

University of Lleida

Gerard Mauri-Capdevila

Hospital Universitari Arnau de Vilanova

Mikel Vicente-Pascual

Hospital Universitari Arnau de Vilanova

Joan Farre

Hospital Universitari Arnau de Vilanova

\section{Research Article}

Keywords: Biomarkers, IL 6, S100b, Stroke, prognosis, volume, acute ischaemic stroke, DWI

Posted Date: December 15th, 2020

DOI: https://doi.org/10.21203/rs.3.rs-119511/v1

License: (c) (i) This work is licensed under a Creative Commons Attribution 4.0 International License.

Read Full License 


\section{Abstract}

Background: The study of biomarkers related to the infarct volume of acute ischaemic stroke (AIS) is a valuable clinical strategy. We conducted a prospective study to evaluate the relationship between a wide panel of biomarkers involved in different biochemical pathways and lesion volume.

Methods: We studied 332 patients with AIS. Infarct volume was calculated from diffusion weighted imaging (DWI). Blood samples were drawn within $24 \mathrm{~h}$ of symptom onset to test a panel of biomarkers that included high-sensitivity C-reactive protein (hs-CRP), IL-6, neuron-specific enolase (NSE), N-terminal pro-B-type natriuretic peptide (NT-ProBNP), S100b, troponin and IL-10.

Results: The median lesion volume was 2.5 cc (IQR: 0.6-15.3). Patients with previous atrial fibrillation, cardioembolic aetiology and total anterior circulation infarct TACl classification had higher lesion volumes than those without them. Patients with previous recent TIA had smaller ischemic lesions than those without it. Age and NIHSS were significantly correlated with lesion volume. In a lineal regression analysis adjusted by aetiology, S100b and IL- 6 emerged as the only biomarkers that could independently predict infarct volume. In contrast, previous recent TIA and small vessel disease were inversely related to infarct volume.

Conclusion: The correlation between the two blood marker levels and ischemic lesion volume would support the use of these biomarkers as a surrogate endpoint in AIS, especially in centres without DWI 24/7. Our findings should be further explored in larger, preferably multicentre studies.

\section{Introduction}

Stroke is a very prevalent disease that always appears amongst the leading causes of death in developed countries $^{1,2}$. It also ranks first amongst diseases that cause disability ${ }^{1,3}$. Since the proposal of a tissular definition of acute ischaemic stroke (AIS) ${ }^{4}$, diffusion weighted imaging (DWI) has become essential for its diagnosis. However, despite its high incidence, not all hospitals that attend AIS patients have access to advanced neuroimaging devices. The possibility of making an approximate diagnostic assessment using biomarkers that can be obtained from a blood analysis is therefore of great interest.

Biomarkers of cerebral tissue damage appear in blood due to the ischemic damage that strokes deal to neurons, myelin and glia; they cause the release of these markers into the blood flow and give us the opportunity to measure them ${ }^{5}$.

We conducted a prospective study to evaluate the relationship between a wide panel of the biomarkers involved in different biochemical pathways. This was done within the first $24 \mathrm{~h}$ after symptom onset in AIS patients, and the lesion volume was recorded.

\section{Results}


A total of 332 patients were included in this study (flow chart in Fig. 1). The mean age was 72.5 (SD 11.7) years old (Table 1). 131 (39.5\%) of the patients were women. Hypertension, which was present in 247 (74.6\%) patients, was the main vascular risk factor. It was followed by dyslipidaemia: 165 (45.9\%) and diabetes mellitus: 105 (29.1\%). 31 (9.3\%) patients had had a transient ischaemic attack (TIA) in the previous 7 days. A cardioembolic aetiology (CE) was detected in $133(36.7 \%)$ patients. The median lesion volume was $2.5 \mathrm{cc}$ (IQR: 0.6-15.3). The biomarker levels are presented in Table 1. 
Table 1

Risk factors, clinical characteristics, previous treatment, episode characteristics and neuroimaging data for the cohort

\section{Variables}

Age, years mean (sd)

Sex women, $\mathrm{n}(\%)$

Alcoholism, n (\%)

Active smoking, $\mathrm{n}(\%)$

Hypertension, $\mathrm{n}(\%)$

Diabetes mellitus, $\mathrm{n}(\%)$

Dyslipidemia, $\mathrm{n}(\%)$

Previous ais, $n(\%)$

Previous tia within 7 days, $\mathrm{n}(\%)$

Atrial fibrillation, $\mathrm{n}(\%)$

Ischaemic heart disease, $\mathrm{n}(\%)$

Previous treatment

Antiplatelet drugs, $\mathrm{n}(\%)$

Anticoagulation, $\mathrm{n}(\%)$

Statins, n (\%)

Renin-angiotensin system blockers, $\mathrm{n}(\%)$

Current stroke study

Stroke code activation, $\mathrm{n}(\%)$

Revascularization therapy, $\mathrm{n}(\%)$

no treatment

rtPA

rtPA plus thrombectomy

thrombectomy
$72.5(11.7)$

$131(39.5)$

23 (7.0) 4 missing

73 (22.1) 1 missing

247 (74.6) 1 missing

92 (27.8) 1 missing

$154(46.4)$

40 (12.0)

31 (9.3)

67 (20.3) 2 missing

37 (11.2) 1 missing

121 (36.4)

$36(10.8)$

126 (38.0)

$171(51.5)$

$166(50)$

$268(82,2)$

53 (16.0)

$10(3.0)$

$1(0.3)$

ItPA, recombinant tissue plasminogen activator; NIHSS, National Institutes of Health Stroke Scale; $P A C l$, partial anterior circulatory infarct; TACl, total anterior circulatory infarct; $P O C l$, posterior circulatory infarction; $L A C l$, lacunar anterior circulatory infarct; $m R S$, modified Rankin Scale; $h s-C R P$, high-sensitivity C-reactive protein; NSE, neuron-specific enolase; NT-ProBNP, N-terminal pro-B-type natriuretic peptide. 


\begin{tabular}{|c|c|}
\hline \multicolumn{2}{|l|}{ Variables } \\
\hline Basal NIHSS: mean (sd) (p25; 50; 75) & $5.9(5.7)(2 ; 4 ; 8)$ \\
\hline Basal mRs: mean (sd) (p25; 50; 75) & $0.46(0.86)(0 ; 0 ; 1)$ \\
\hline Aetiology, n (\%) & $59(17.8)$ \\
\hline atheromatous & $126(38.0)$ \\
\hline cardioembolism & $63(19.0)$ \\
\hline small vessel & $77(23.2)$ \\
\hline indeterminate & $7(2.1)$ \\
\hline \multicolumn{2}{|l|}{ unusual } \\
\hline Oxfordshire classification, n (\%) & 8 missing \\
\hline $\mathrm{TACl}$ & $53(16.4)$ \\
\hline $\mathrm{PACl}$ & $126(38.9)$ \\
\hline $\mathrm{POCl}$ & $38(11.7)$ \\
\hline LACl & $107(33.0)$ \\
\hline Total stroke volume: & $18.3(41.0)(0.56 ; 2.53 ; 15.28)$ \\
\hline \multicolumn{2}{|l|}{ mean $(\mathrm{sd})(\mathrm{p} 25 ; 50 ; 75)$} \\
\hline \multicolumn{2}{|l|}{ Biomarkers: (p25; 50; 75) } \\
\hline NSE pg/ml & $(9.4 ; 11.4 ; 16)$ \\
\hline IL6 pg/ml & $(4.3 ; 8 ; 14.9)$ \\
\hline NT-ProBnP pg/ml & $(120.6 ; 402.3 ; 1281.8)$ \\
\hline S100b pg/ml & $(0.1 ; 34.8 ; 70.0)$ \\
\hline hs CRP & $(1.8 ; 4.2 ; 11.9)$ \\
\hline hs Troponin pg/ml & $(7.3 ; 13.2 ; 24.4)$ \\
\hline IL 10 pg/ml & $(1.0 ; 1.0 ; 2.5)$ \\
\hline \multicolumn{2}{|c|}{$\begin{array}{l}\text { rtPA, recombinant tissue plasminogen activator; NIHSS, National Institutes of Health Stroke Scale; } \\
\text { PACI, partial anterior circulatory infarct; TACl, total anterior circulatory infarct; POCl, posterior } \\
\text { circulatory infarction; LACl, lacunar anterior circulatory infarct; } m R S \text {, modified Rankin Scale; } h \text { s-CRP, } \\
\text { high-sensitivity C-reactive protein; NSE, neuron-specific enolase; NT-ProBNP, N-terminal pro-B-type } \\
\text { natriuretic peptide. }\end{array}$} \\
\hline
\end{tabular}

We observed that patients with previous cases of atrial fibrillation, CE and TACl classification had higher lesion volumes than those without them. In contrast, patients with previous recent TIA had smaller 
lesions than those who did not (Table 2). Age and NIHSS were also significantly correlated with lesion volume (Spearman's coefficient: $0.18, p=0.001$ and $0.45, p<0.001$, respectively).

Table 2

Univariate analyses of variables associated with lesion volume

\begin{tabular}{|c|c|c|c|}
\hline Variable & No & YEs & $\mathrm{p}$ value \\
\hline Sex women & $2.3(0.5-13.7)$ & $5.5(0.6-24.0)$ & 0.075 \\
\hline Alcoholism & $2.6(0.5-17.0)$ & $1.4(0.6-4.9)$ & 0.401 \\
\hline Active smoking & $2.6(0.5-17.0)$ & $1.9(0.4-15.2)$ & 0.244 \\
\hline Hypertension & $1.5(0.4-15.2)$ & $3.0(0.6-16.0)$ & 0.102 \\
\hline Diabetes mellitus & $2.5(0.6-15.2)$ & $2.6(0.5-14.1)$ & 0.692 \\
\hline Dyslipidemia & $2.8(0.6-19.6)$ & $2.3(0.5-14.3)$ & 0.186 \\
\hline Previous AIS & $2.4(0.5-15.4)$ & $3.9(0.6-22.3)$ & 0.677 \\
\hline Previous TIA within 7 days & $2.8(0.6-17.4)$ & $0.7(0.2-8.2)$ & 0.007 \\
\hline Previous atrial fibrillation & $1.9(0.5-12.9)$ & $9.1(1.7-30.0)$ & $<0.001$ \\
\hline Ischemic heart disease & $2.5(0.6-16.0)$ & $2.6(0.5-16.3)$ & 0.720 \\
\hline Etiology: & & $5.7(0.7-20.4)$ & $<0.001$ \\
\hline Atheromatous & & $8.7(1.5-30.0)$ & \\
\hline Cardioembolism & & $0.4(0.2-0.8)$ & \\
\hline Small vessel & & $2.8(0.7-15.2)$ & \\
\hline Indeterminate & & $1.5(1.1-37.2)$ & \\
\hline \multicolumn{4}{|l|}{ Unusual } \\
\hline \multicolumn{2}{|l|}{ Oxfordshire classification } & $35.8(9.3-133.7)$ & $<0.001$ \\
\hline \multicolumn{2}{|l|}{$\mathrm{TACl}$} & $4.7(0.8-18.3)$ & \\
\hline \multicolumn{2}{|l|}{ PACl } & $1.8(0.4-7.3)$ & \\
\hline \multicolumn{2}{|l|}{$\mathrm{POCl}$} & $0.7(0.2-2.3)$ & \\
\hline \multicolumn{4}{|l|}{ LACl } \\
\hline \multicolumn{4}{|c|}{ Volume unit: Cubic Centimetre } \\
\hline \multicolumn{4}{|c|}{$\begin{array}{l}\text { PACl, partial anterior circulatory infarct; TACl, total anterior circulatory infarct; POCl, posterior } \\
\text { circulatory infarction; } L A C l \text {, lacunar anterior circulatory infarct; mRS, modified Rankin Scale; TIA, } \\
\text { transient ischemic attack; AIS, acute ischemic stroke. }\end{array}$} \\
\hline
\end{tabular}


All the biomarker levels were significantly correlated with infarct volume (Table 3); S100b exhibited the strongest correlation. As a consequence of the relationship between etiological subtypes and infarct volume, we also analysed biomarker levels across aetiology (Table 4 and Fig. 2). NSE, IL6, NT-ProBNP, $\mathrm{S} 100 \mathrm{~b}$ and troponin levels were higher in CE than in the other subtypes. IL10 levels were higher in cases of unusual aetiology than in others.

Table 3

Biomarker correlations with infarct size

\begin{tabular}{|lll|}
\hline Biomarker & Spearman's coefficient & P value \\
\hline NSE & 0.191 & $<0.001$ \\
\hline IL-6 & 0.373 & $<0.001$ \\
\hline NT-ProBNP & 0.271 & $<0.001$ \\
\hline S100b & 0.484 & $<0.001$ \\
\hline CRP & 0.202 & $<0.001$ \\
\hline Troponin & 0.193 & $<0.001$ \\
\hline IL-10 & 0.161 & 0.003 \\
\hline $\begin{array}{l}\text { hs-CRP, high-sensitivity C-reactive protein; NSE, neuron-specific enolase; NT-ProBNP, N-terminal pro-B- } \\
\text { type natriuretic peptide. }\end{array}$ & \\
\hline
\end{tabular}


Table 4

Biomarker levels by aetiologic subtype

\begin{tabular}{|c|c|c|c|c|c|c|}
\hline \multirow[t]{2}{*}{ Biomarker } & \multicolumn{5}{|l|}{ Aetiology } & \multirow[t]{2}{*}{$p$} \\
\hline & Atheromatous & $\begin{array}{l}\text { Cardio- } \\
\text { embolic }\end{array}$ & $\begin{array}{l}\text { Small } \\
\text { vessel }\end{array}$ & Indeterminate & Unusual & \\
\hline$N S E$ & $11.4(9.2-14)$ & $\begin{array}{l}12.8(9.7- \\
18.6)\end{array}$ & $\begin{array}{l}10.4(8.9- \\
13.7)\end{array}$ & $\begin{array}{l}11.5(9.4- \\
16.5)\end{array}$ & $\begin{array}{l}10.8(9.4- \\
23.4)\end{array}$ & 0.012 \\
\hline IL-6 & $8.9(4.9-14.6)$ & $\begin{array}{l}10.9(6.1- \\
23.4)\end{array}$ & $4.8(2.8-9.7)$ & $8.1(5.4-14.7)$ & $\begin{array}{l}4.5(1.9- \\
10.0)\end{array}$ & $<.001$ \\
\hline $\begin{array}{l}\text { NT- } \\
\text { ProBNP }\end{array}$ & $215(89-627)$ & $\begin{array}{l}1194(468- \\
2274)\end{array}$ & $\begin{array}{l}110(61- \\
333)\end{array}$ & $\begin{array}{l}460(135- \\
1239)\end{array}$ & $\begin{array}{l}60(14- \\
329)\end{array}$ & <. \\
\hline S100b & $\begin{array}{l}66(38.3- \\
96.8)\end{array}$ & $83(54-140.4)$ & $\begin{array}{l}42.2(33.9- \\
60)\end{array}$ & $\begin{array}{l}69(42.3- \\
136)\end{array}$ & $\begin{array}{l}46.1(30.9- \\
103)\end{array}$ & $<.001$ \\
\hline hs CRP & $4.4(1.6-13.5)$ & $\begin{array}{l}6.7(2.6- \\
18.1)\end{array}$ & $2.4(1.5-5.8)$ & $3.4(1.7-7)$ & $\begin{array}{l}1.8(1.2- \\
13.7)\end{array}$ & $\begin{array}{l}< \\
0.001\end{array}$ \\
\hline $\begin{array}{l}\text { hs } \\
\text { troponin }\end{array}$ & $13(7.7-21.9)$ & $\begin{array}{l}18.2(10.9- \\
32.2\end{array}$ & $\begin{array}{l}8.6(4.9- \\
13.8)\end{array}$ & $\begin{array}{l}17.2(9.1- \\
35.4\end{array}$ & $\begin{array}{l}4.2(3.5- \\
10.6)\end{array}$ & $\begin{array}{l}< \\
0.001\end{array}$ \\
\hline IL-10 & $\begin{array}{l}0.98(0.98- \\
2.64)\end{array}$ & $\begin{array}{l}1.56(0.98- \\
3.18)\end{array}$ & $\begin{array}{l}0.98(0.98- \\
1.80)\end{array}$ & $\begin{array}{l}0.98(0.98- \\
2.44)\end{array}$ & $\begin{array}{l}3.24(1.60- \\
4.68)\end{array}$ & 0.002 \\
\hline
\end{tabular}

Finally, in a lineal regression analysis that was adjusted by aetiology (Table 5), S100b and IL-6 emerged as the only biomarkers that could independently predict the infarct volume. In contrast, previous recent TIA and small vessel disease were inversely related to infarct volume. 
Table 5

Predictors of infarct volume

\begin{tabular}{|lllll|}
\hline Variables & Beta Coefficient & \multicolumn{2}{c|}{ Beta 95\% Cl } & \\
\cline { 3 - 5 } & & Inferior limit & Superior limit & \\
\hline S100b & 0.401 & 0.253 & 0.550 & $<0.001$ \\
\hline IL-6 & 0.186 & 0.042 & 0.329 & 0.011 \\
\hline Small vessel aetiology & -1.781 & -2.278 & -1.284 & $<0.001$ \\
\hline Indeterminate aetiology & -0.587 & -1.021 & -0.153 & 0.008 \\
\hline Previous recent TIA & -0.649 & -1.259 & -0.039 & 0.037 \\
\hline NIHSS & 0.064 & 0.013 & 0.115 & 0.014 \\
\hline TACI & 0.945 & 0.190 & 1.699 & 0.014 \\
\hline Age & -0.023 & -0.040 & -0.007 & 0.006 \\
\hline $\begin{array}{l}\text { NIHSS, National Institutes of Health Stroke Scale; TACl, total anterior circulatory infarct; POCl, posterior } \\
\text { circulatory infarction; TIA, transient ischemic attack }\end{array}$ & & \\
\hline
\end{tabular}

\section{Discussion}

Our prospective study evaluated the correlation of a panel of different biomarkers with infarct size. We combined biomarkers of inflammation, atrial dysfunction and neuronal and glial dysfunction ${ }^{5-7}$ and observed that IL-6 and S100b levels were able to independently predict infarct volume. In contrast to the other biomarkers, their correlation remained unchanged after the multivariate analyses adjusted for stroke aetiology. These findings have clinical relevance because the level of these markers in blood may indicate the extension of brain infarction without the need to use neuroimaging techniques. Some previous studies identified the correlation between S100b and the size of the infarction and/or the prognosis in AIS ${ }^{5,8-10}$. S100 b is detected in glial cells of the central nervous system, including:

oligodendrocytes, ependymocytes, astrocytes, and neuronal progenitor cells, amongst others. ${ }^{12}$ It can be found in both CSF and blood serum in different types of brain disease, such as: brain tumours, neuroinflammatory and neurodegenerative disorders, psychiatric disorders, cerebral infections, subarachnoid haemorrhage, acute brain injury and cerebral infarction ${ }^{8,11}$. The peak value is observed 24 hours after $\mathrm{AIS}^{10}$. According to systematic revision measurements, S100b readings taken more than $24 \mathrm{~h}$ after AIS are better correlated with subacute infarct size than measurements taken earlier ${ }^{5}$. We studied the correlation of basal levels with infarct size because management decisions must be taken during the acute phase of ischemic stroke. There is, however, less previous evidence available about the utility of IL6 associated with stroke. A recent meta-analysis of 24 studies did, however, confirm an otherwise unlikely translation of IL-6 into clinical practice for the prognosis of AIS ${ }^{12}$. IL-6 is an inflammatory cytokine that is upregulated by cerebral ischemia ${ }^{13}$. Some studies, which included relatively few patients, described an 
association between peak IL-6 levels during the first week after AIS and lesion volume ${ }^{14}$. However, as in the case of the previous biomarker, the relationship between basal levels and the neuroimaging endpoint was of clinical interest. In addition, basal IL-6 levels seemed to be less influenced by complications related to worse outcomes than to increases in acute phase reactants, like infectious ${ }^{15}$.

The main limitation of the study was its size. However, we consider that the study population may be representative of the general Spanish population because the clinical characteristics were similar to those of other larger cohorts ${ }^{16}$. Furthermore, the clinical variables related to infarct size had previously been described as $\mathrm{CE}^{17}$, TACl and stroke severity. We also wish to highlight how previous recent TIA was associated with small infarcts, as in previously reported studies ${ }^{18}$. This could be a sign of ischaemic tolerance induced by episodes of transient ischaemia prior to the definitive stroke ${ }^{19}$.

\section{Materials And Methods}

We carried out a single-centre, registry-based, cohort study that included AIS patients from the Hospital Universitari Arnau de Vilanova, in Lleida, Spain. All the patients studied were subjected to DWI within a week of the onset of symptoms. Patients without clear evidence of acute ischaemic lesion in neuroimaging were excluded from the study, following AHA/ASA guidelines ${ }^{4}$. All the patients were attended to in the emergency room, by a specialist neurologist, between January 2015 and January 2017. The study was approved by the local ethics committee (comité ètic de l'Hospital Universitari Arnau de Vilanova de Lleida, Lleida. Spain), and all patients provided written informed consent. The study was performed in accordance with the relevant guidelines and regulations including Declaration of Helsinki.

Patients were classified etiologically according to TOAST criteria (Trial of Org 10,172 in Acute Stroke Treatment) as large-artery occlusive disease, small-vessel disease, cardioembolic, other cause, or undetermined cause. The following patient's characteristics were collected: age at admission, gender, vascular risk factors, prior treatments, revascularization therapies (recombinant tissue plasminogen activator: rt-PA; thrombectomy; rt-PA and thrombectomy) premorbid modified Rankin scale (mRS). We used the clinical topographic classification of Oxfordshire community stroke project criteria ${ }^{20}$ to classify patient symptoms into $\mathrm{PACl}$, partial anterior circulatory infarct; $\mathrm{TACl}$, total anterior circulatory infarct; $\mathrm{POCl}$, posterior circulatory infarction; and LACI, lacunar anterior circulatory infarct.

Blood samples were drawn on arrival at the emergency department, and within $24 \mathrm{~h}$ of symptom onset, to test a panel of biomarkers that included: high-sensitivity C-reactive protein (hs-CRP), IL-6, neuron-specific enolase, (NSE), N-terminal pro-B-type natriuretic peptide (NT-ProBNP), S100b, high-sensitivity troponin and IL-10. Blood was drawn into EDTA tubes and centrifuged at $1150 \mathrm{~g}$ for $15 \mathrm{~min}$; the resulting plasma was then aliquoted into cryovials for immediate storage at at $-80^{\circ} \mathrm{C}$ until analysis (Plataforma Biobancos PT17/0015/0027) ${ }^{7}$. NSE, IL-6, S100b, hs troponin and NT-ProBNP were assayed in serum using a commercially available electrochemiluminescence immunoassay based on a polyclonal antibody-based sandwich chemiluminescence assay (Roche Diagnostics, Grenzach, Germany) using an autoanalyzer 
(Elecsys, Olathe, KS, USA). hsCRP was measured using a solid-phase two-site chemiluminescent immunometric assay on the immunoassay system IMMULITE system (Siemens Healthcare, Erlangen, Germany). IL-1 and IL 10 were quantified in serum, using a solid-phase sandwich enzyme-linked immune sorbent assay (ELISA), which is commercially available from BIONOVA. The absorbance was read in a spectrophotometer using a wave length of $450 \mathrm{~nm}$.

Positive DWI was defined as areas of high signal intensity on isotropic DWI, which reflected acute ischemic lesion. One Neuroradiologist, who was blinded to the clinical procedure, established the presence or absence of DWI abnormalities. OsiriX V.4.0 imaging software ${ }^{21}$ was also used to calculate the total volume of DWI abnormality. We manually outlined any abnormalities using the OsiriX closedpolygon tool to identify any regions of interest (ROI). Any ROls located between segmented slices were automatically interpolated. We then determined the resulting DWI abnormality volume.

Data were reported using standard descriptive statistics. Univariate analysis was performed to detect variables associated with the DWI volume. The Kruskal-Wallis test was used to find any significant differences in biomarker levels between different aetiology groups. Spearman's correlation coefficient was used to demonstrate the association between the different biomarkers and the stroke volume. As the biomarkers and the volume were not normally distributed, we divided each biomarker into quintiles and measured the distribution or mean value (confidence interval) of the volume in each quintile in order to visualize them graphically. Finally, a multivariate linear regression was conducted for variables that met $p$-value $<0.1$ on the univariate analysis. A probability value of $<0.05$ was considered significant.

\section{Declarations}

Author Contributions: JF and FP conceived the study. JF and FP designed experiments. GMC, MVP and JFR cohorts' recruitment, clinical data and blood samples acquisition. FP neuroimaging analysis. JF sample processing and data analysis. JFR and FP participated on data interpretation and draft the manuscript. All authors critically revised the final version of the manuscript. All authors approved the final version to be published. FP procured funding.

Funding: This study was supported by the Government of Catalonia-Agència de Gestió d'Ajuts Universitaris i de Recerca (FP: 2017 SGR 1628) Instituto de Salud Carlos III and co-funded by European Union (ERDF/ESF, "Investing in your future" and "A way to build Europe") (FP: PI17-01725, PI14/01574) and the INVICTUS plus Research Network (Carlos III Health Institute) (FP: RD16-0019-0017).

Acknowledgments: We are grateful to all recruited patients, as well as the members of Clinical Neuroscience group at IRBLleida, personnel of Neurology Department at Hospital Universitari Arnau de Vilanova de Lleida for scientific discussions and instrumental help, and Mr. Manuel Quintana for statistical help. We also acknowledge the use of the facility of Biobank (B.0000682) at IRBLleida and Plataforma Biobancos PT17/0015/0027.

Conflicts of Interest: The authors declare no conflict of interest. 


\section{References}

1 Feigin, V. L., Norrving, B. \& Mensah, G. A. Global Burden of Stroke. Circ Res 120, 439-448, doi:10.1161/CIRCRESAHA.116.308413 (2017).

2 Mozaffarian, D. et al. Heart Disease and Stroke Statistics-2016 Update: A Report From the American Heart Association. Circulation 133, e38-e360, doi:10.1161/CIR.0000000000000350 (2016).

3 Feigin, V. L. et al. Global and regional burden of stroke during 1990-2010: findings from the Global Burden of Disease Study 2010. Lancet 383, 245-254 (2014).

4 Easton, J. D. et al. Definition and evaluation of transient ischemic attack: a scientific statement for healthcare professionals from the American Heart Association/American Stroke Association Stroke Council; Council on Cardiovascular Surgery and Anesthesia; Council on Cardiovascular Radiology and Intervention; Council on Cardiovascular Nursing; and the Interdisciplinary Council on Peripheral Vascular Disease. The American Academy of Neurology affirms the value of this statement as an educational tool for neurologists. Stroke 40, 2276-2293, doi:10.1161/strokeaha.108.192218 (2009).

5 Ahmad, O., Wardlaw, J. \& Whiteley, W. N. Correlation of levels of neuronal and glial markers with radiological measures of infarct volume in ischaemic stroke: a systematic review. Cerebrovasc Dis 33, 4754, doi:10.1159/000332810 (2012).

6 Purroy, F. et al. The determination of copeptin levels helps management decisions among transient ischaemic attack patients. Acta Neurol Scand, doi:10.1111/ane.12523 (2015).

7 Purroy, F. et al. N-terminal pro-brain natriuretic peptide level determined at different times identifies transient ischaemic attack patients with atrial fibrillation. European journal of neurology 21, 679-683, doi:10.1111/ene.12222 (2014).

$8 \mathrm{Ye}, \mathrm{H}$. et al. Serum S100B levels may be associated with cerebral infarction: a meta-analysis. $J$ Neurol Sci 348, 81-88, doi:10.1016/j.jns.2014.11.010 (2015).

9 Selcuk, O. et al. The Relationship of Serum S100B Levels with Infarction Size and Clinical Outcome in Acute Ischemic Stroke Patients. Noro Psikiyatr Ars 51, 395-400, doi:10.5152/npa.2014.7213 (2014).

10 Brea, D. et al. Temporal profile and clinical significance of serum neuron-specific enolase and S100 in ischemic and hemorrhagic stroke. Clin Chem Lab Med 47, 1513-1518, doi:10.1515/CCLM.2009.337 (2009).

11 Zurek, J. \& Fedora, M. The usefulness of S100B, NSE, GFAP, NF-H, secretagogin and Hsp70 as a predictive biomarker of outcome in children with traumatic brain injury. Acta Neurochir (Wien) 154, 93103; discussion 103, doi:10.1007/s00701-011-1175-2 (2012). 
12 Bustamante, A. et al. Prognostic value of plasma chitotriosidase activity in acute stroke patients. Int J Stroke 9, 910-916, doi:10.1111/ijs.12160 (2014).

13 Bustamante, A. et al. Prognostic value of blood interleukin-6 in the prediction of functional outcome after stroke: a systematic review and meta-analysis. Journal of neuroimmunology 274, 215-224, doi:10.1016/j.jneuroim.2014.07.015 (2014).

14 Smith, C. J. et al. Peak plasma interleukin-6 and other peripheral markers of inflammation in the first week of ischaemic stroke correlate with brain infarct volume, stroke severity and long-term outcome. BMC Neurol 4, 2, doi:10.1186/1471-2377-4-2 (2004).

15 Wartenberg, K. E. et al. Infection after acute ischemic stroke: risk factors, biomarkers, and outcome. Stroke Res Treat 2011, 830614, doi:10.4061/2011/830614 (2011).

16 Purroy, F. et al. Age- and Sex-Specific Risk Profiles and In-Hospital Mortality in 13,932 Spanish Stroke Patients. Cerebrovascular diseases (2019).

17 Ferro, J. M. Cardioembolic stroke: an update. Lancet Neuro/ 2, 177-188 (2003).

18 Wegener, S. et al. Transient ischemic attacks before ischemic stroke: preconditioning the human brain? A multicenter magnetic resonance imaging study. Stroke 35, 616-621, doi:10.1161/01.STR.0000115767.17923.6A01.STR.0000115767.17923.6A [pii] (2004).

19 ladecola, C. \& Anrather, J. Stroke research at a crossroad: asking the brain for directions. Nat Neurosci 14, 1363-1368, doi:10.1038/nn.2953 (2011).

20 Bamford, J., Sandercock, P., Dennis, M., Burn, J. \& Warlow, C. Classification and natural history of clinically identifiable subtypes of cerebral infarction. Lancet 337, 1521-1526, doi:10.1016/01406736(91)93206-o (1991).

21 Rosset, A., Spadola, L. \& Ratib, O. OsiriX: an open-source software for navigating in multidimensional DICOM images. Journal of digital imaging 17, 205-216, doi:10.1007/s10278-004-10146 (2004).

\section{Figures}




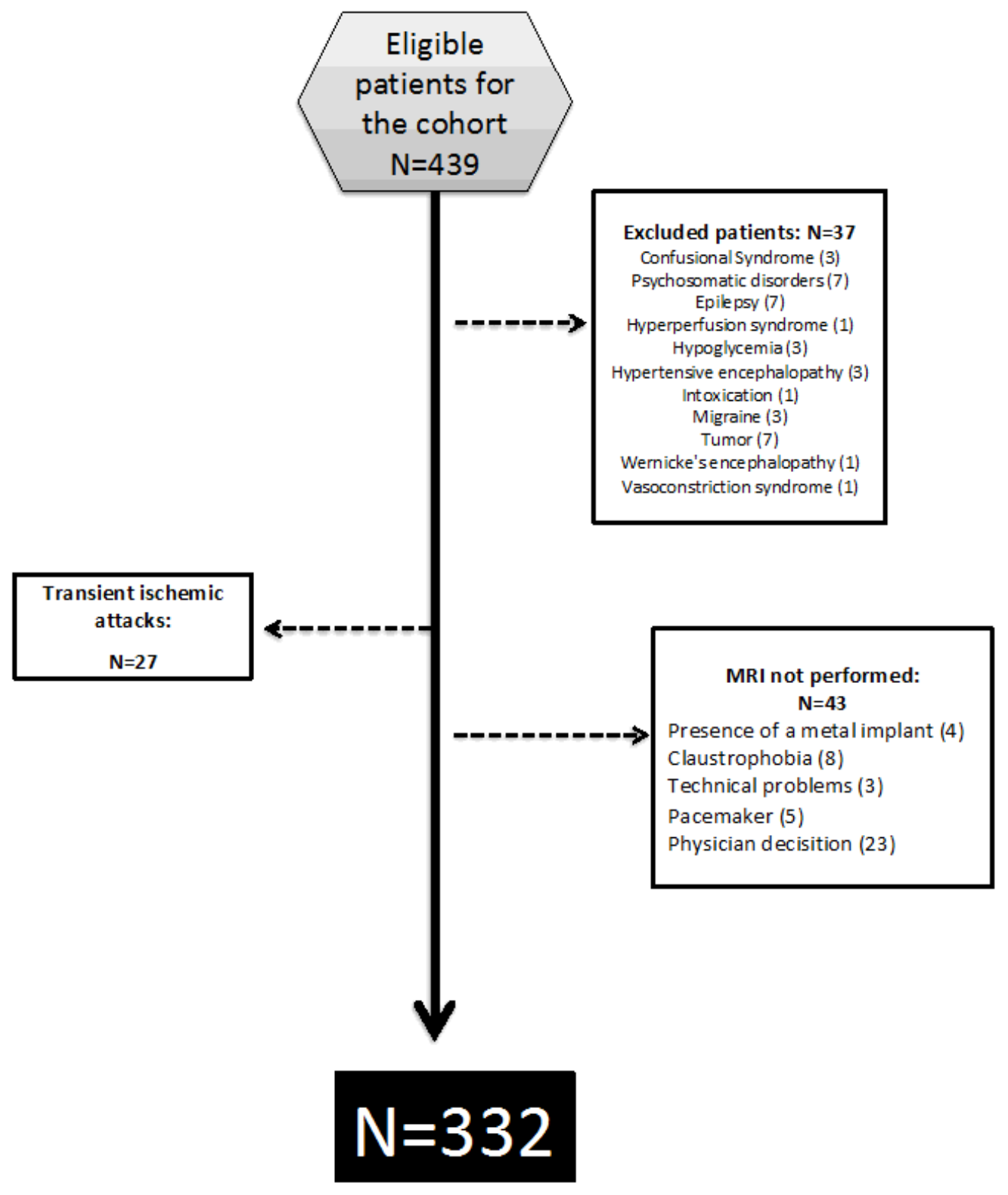

\section{Figure 1}

Flow chart of the inclusion of patients 

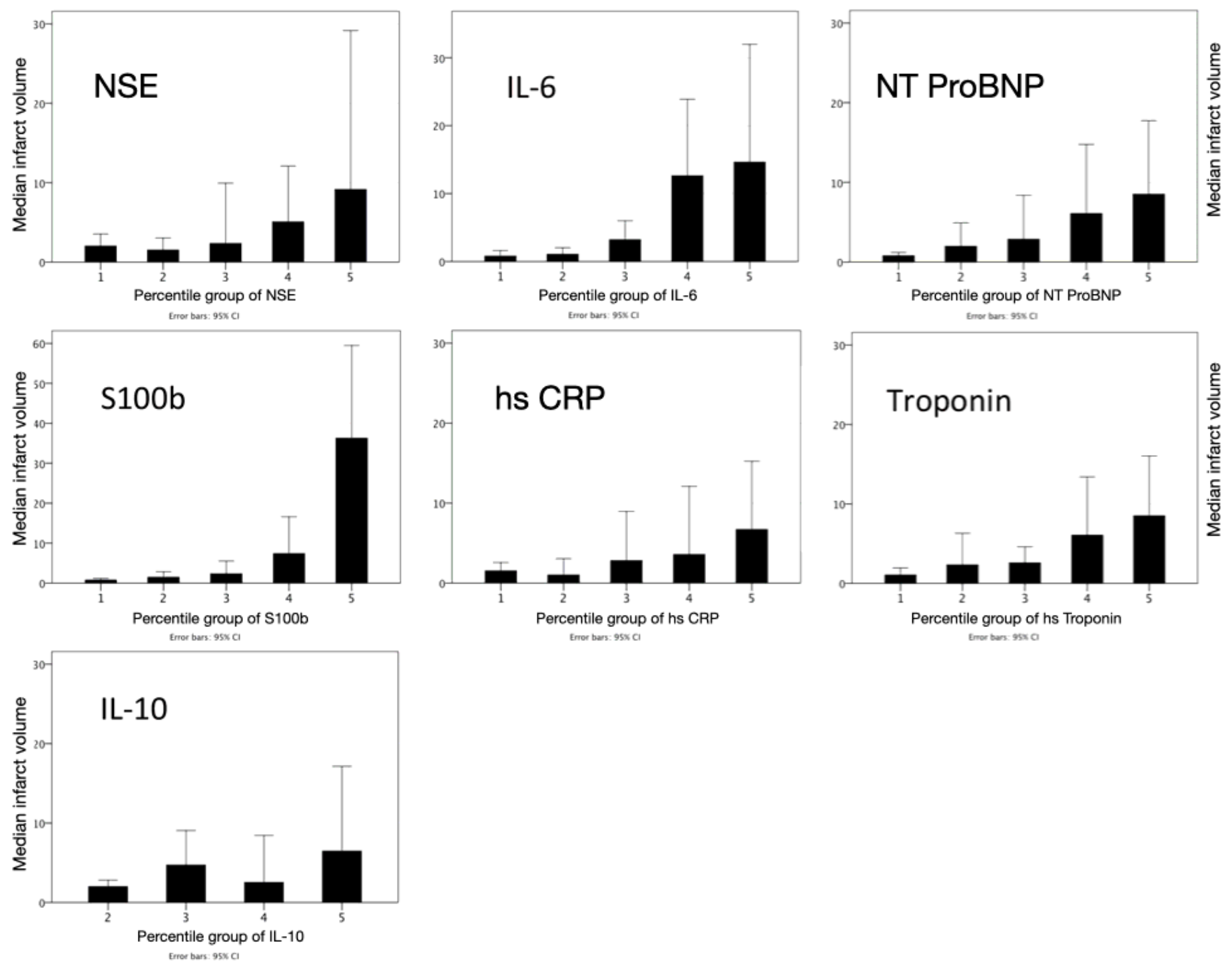

Figure 2

Correlation between biomarker levels and infarct volume 